\title{
A priority-based adaptive scheme for multi-view live streaming over HTTP
}

\author{
Weizhan Zhang, Shuyan Ye, Bin Li, Hui Zhao, Qinghua Zheng \\ MOEKLINNS Lab, Department of Computer Science and Technology, Xi'an Jiaotong University \\ No.28, Xianning West Road, Xi'an, 710049, China \\ Telephone number \&5 Fax number: 008629-82663860 \\ Email address: zhangwzh@mail.xjtu.edu.cn
}

\begin{abstract}
Adaptive streaming over Hypertext Transport Protocol (HTTP) has been widely used for the transmission of video content. Most of the existing studies about HTTP adaptive streaming (HAS) concentrate on improving resource efficiency, fairness among users, and quality of service for single-source videos. However, there is a growing number of live streaming applications, such as video-casts of courses, where service providers want to transmit video streams of different scenes simultaneously over the bandwidth-constrained network. Since concurrent streams further deteriorate the insufficient bandwidth situation, a systematic solution is highly needed to utilize the resources efficiently and guarantee the quality of multi-view video services. In this paper, we propose a priority-based adaptive scheme for multi-view live streaming based on HTTP streaming. Firstly, we adopt an integrated bandwidth prediction approach to calculate the available bandwidth, solving the low bandwidth utilization problem existing in the benchmark method. Secondly, we design a unified segment request strategy to restrict the interval between each request, and guarantee the synchronization of different live video streams. Finally, an adaptive scheduling algorithm is proposed with respect to priorities, to dynamically adjust the quality level of multiple video streams and improve the quality of multi-view video service. Simulations show that the proposed scheme makes considerable improvements compared to the benchmarks.
\end{abstract}

Keywords: HTTP adaptive streaming; priority-based; Multi-view; Bandwidth utilization.

\section{Introduction}

On account of the abundance of Web platforms and broadband connections, multimedia content has been widely supported in many web applications by a variety of streaming technologies such as HTTP Adaptive Streaming (HAS) [1]. Those acceleration techniques are of great importance to meet the new challenges for media content delivery, such as the cloud-based CDNs[2], WAN acceleration techniques[3], CDNs in mobile networks[4] and so on. Since the variability of bandwidth conditions and CDNs environments complicates the provisioning of resources along the path from the video source to the client side, the dynamical adaptability over HTTP adaptive streaming becomes a competitive character and receives increasing attention from many researchers[5].

In HAS, one media stream is divided into multiple segments and each segment is encoded into different quality levels. The client (or receiver) can request the appropriate segment in the adaptation set according to the information (e.g., bit rate) available, and thus support the flexibility of the network condition. In order to improve the resource efficiency and quality of service, most studies about HAS are focused on resource prediction and rate adaptation in single-source video streaming. However, in many video applications, such as 3D entertainment or live lecture broadcasting, an increasing number of service providers want to transmit concurrent multiple videos of one scene simultaneously [6-8]. The service provider tries to obtain the ability that a user can simultaneously watch multiple videos on one screen by a client. In particular, the motivation of this research comes from integrating multi-view HAS in an e-learning system. The system is designed to deliver multiple time-related views of a live lecture spot, such as the camera video of teacher, the camera video of students, and the screen video of teacher's computer. Taking advantage of the existing method for single-source videos and treating these streams independently cannot guarantee the synchronicity among concurrent video streams. 
Therefore, we put forward a priority-based adaptive scheme for multi-view live streaming based on HTTP, which can provide concurrent multiple videos presented in one scene of a client, and can guarantee differentiated service for different views with high resource utilization. Firstly, we adopt an integrated bandwidth prediction approach. It utilizes the unified measurement to predict the available bandwidth. Thus, the client can obtain a higher predicted bandwidth, which can lead to a higher requested bit rate by the clients. Hence, it can improve bandwidth utilization in the method that treats multiple video streams independently. Secondly, we design a unified segment request strategy for multiview video streaming. In this strategy, each request in the current transmission cycle is compelled to wait until all the video segments in the last transmission cycle have been completed. The proposed unified segment request strategy can decide the time when requests can get their appropriate video segments and restrict the interval between requests, thus, segments from different live video streams in the same scenario can be requested and played synchronously, which means that all the video streams of one scene can be requested and then played synchronized in timing. Finally, we propose an adaptive scheduling algorithm for multi-view video streaming with respect to priorities. Without loss of generality, we define the importance or the priorities of a video stream in accordance with their bit rates. For instance , in distance education system, there are two video streams in the same web page, one is the video of camera and the other is the video of screen. The designers usually offer a larger size(higher bit rate) to the video stream of screen which contain more important information, so the video stream of screen gets a higher priority. Notice that our algorithm can guarantee differentiated service for different views according to their priorities. Besides the priority allocation strategy adopted in this paper, the different allocation strategies will also be accepted. Considering each video stream has more than one quality level, we assume that the stream with a higher average bit rate obtains a higher priority. When the bandwidth decreases and becomes insufficient, the quality level of the lower priority stream will decrease first. On the contrary, the higher priority video stream will first increase to a higher quality level. Thus, this algorithm guarantees differentiated service for different views with high resource utilization for the multi-view video service under limited and varying bandwidth conditions.

This paper is organized as follows. Section 2 gives an overview of the related work on resource prediction and rate adaptation in HAS. The architecture of the HTTP adaptive system for multi-view live streaming is shown in Section 3. Our priority-based adaptive scheme is presented in Section 4. Experiments are presented in Section 5. Finally, Section 6 draws conclusions.

\section{Related Work}

Most studies on HTTP adaptive streaming focus on optimizing the performance of several basic aspects in adaptive streaming, such as bandwidth prediction, rate prediction, and rate adaptation.

The bandwidth prediction approach is the pre-condition for the scheme proposed in this paper. To address the resource instability of networks, some researchers focus on predicting the available bandwidth so as to dynamically adjust the quality level of video segments. For instance, Thang et al put forward a prediction algorithm based on previous segment throughputs[9]. It is very easy to calculate the segment throughput by dividing the segment data size via the request-response duration, ranging from the instant of sending the request to the instant of receiving the last byte of the response. However, when the segment duration is long (e.g., 10s), the preceding method is not suitable to represent the instant fluctuations of the bandwidth. Another prediction algorithm is based on download throughput samples[10], it estimates the available transmission bandwidth of the next slice according to the average HTTP/TCP throughput of last segment. We have improved this method by dividing the segment into very short intervals, the accuracy of this algorithm is improved. The new method has no relationship with the length of each video segment and we have utilized it in our previous work[11].

Metadata obtained by the client side already has information about the bit rate of each segment. However, in the case of Variable Bit Rate (VBR) case, the instant bit rate could be much higher than the specified bit rate of video segments. So it seems necessary to select another segment in the same time interval if the instance bit rate of the active one is estimated to be higher than the available bandwidth. Thang et al propose a bit rate prediction in [10] and their experiments show that it can be easily computed by obtaining some sample segments. However, most online video streaming are not real-time video techniques, they are often willing to tolerate a playback delay of several seconds or minutes in exchange for a smaller bandwidth requirement. Taking advantage of such a sensitive method may lead to unnecessary switches in quality levels. 
Some other researches aim at scheduling video segments of different rates to maintain the quality of service (QoS) while coping with varying bandwidths. Jiang et al introduce immediate download and periodic download in their HTTPbased adaptive streaming research[12]. They improve the periodic download method and provide a new way to schedule the video segments. Besides, a scheme that can control the quality of service is designed by Son et al[13]. The relative one-way delay trend is used to determine whether the required bandwidth is available in this scheme. It can improve the service quality effectively. Yunqiang Liu et al propose a segment-based patching scheme which allocates the transmission resource adaptively according to varying client request rates[14]. They claim that their technique can smoothly adjust itself to cope with the newly updated request rate so as to minimize the bandwidth requirement. However, all the methods discussed above focus on single-source videos, and they exhibit poor performance in the multi-view video streaming according to our experiments.

In this paper, we focus on guaranteeing synchronous streaming for multiple video streams presented in the same scene, and concentrate on providing differentiated high-quality service with high resource utilization, which is not addressed by the existing approaches in literature.

\section{Architecture of HTTP Adaptive System for Multi-View Live Streaming}

As shown in Fig.1, the HTTP adaptive system for multi-view live streaming consists of four parts: data collector, web server, distributed components, and client side.

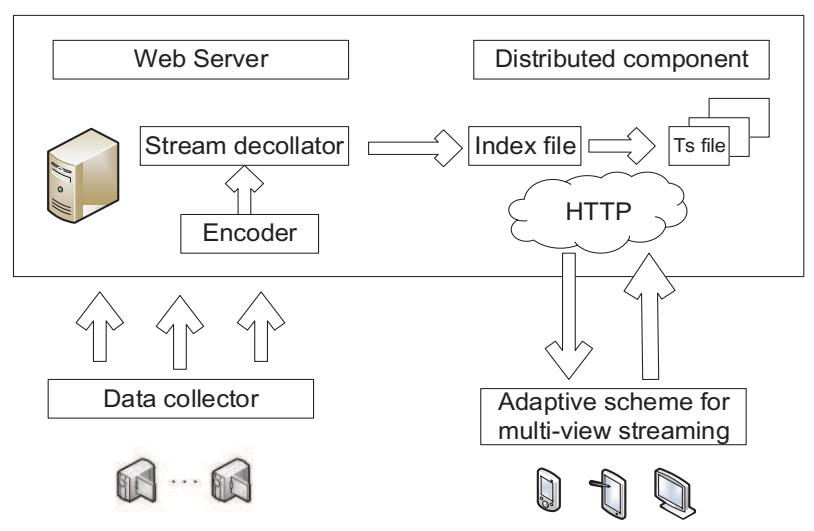

Figure 1: The HTTP adaptive system for multi-view live streaming

Firstly, the data collector collects the video data from different scenes and sends them to the web server. Then the web server encodes the original data into streams and encapsulates them into a standard codec format by the deployed encoder. It also divides each encoded stream into segments (i.e., ts files) with different quality levels according to certain rules by the specific decollator.

At the same time, the distributed component generates the index file (i.e., m3u8 file) that points to each segment. In the case of live streaming, the index file can be considered as a sliding window with video playlists as shown in Fig.2. When a new segment has been produced, information in the index file is also updated, the Uniform Resource Identifier (URI) of the new segment is added to the end of the window, and the oldest one is deleted. Thus, there is always a fixed number of recent URIs in the window. 


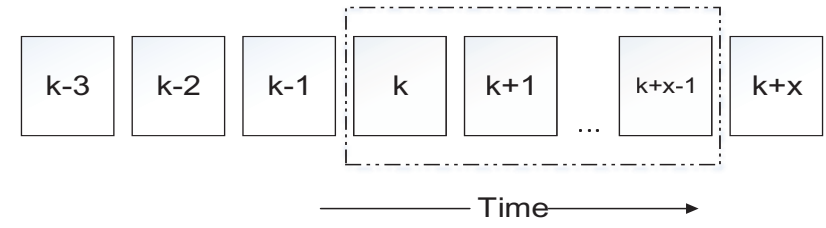

Figure 2: Sliding window playlist

Then, the client side can issue the GET request for the index file and obtain appropriate segments by using our adaptive scheme which will be interpreted in Section 4.

\section{Priority-Based Adaptive Scheme for Multi-View Live Streaming}

Since the adaptive algorithm should be designed according to the network condition, available bandwidth is the principal information in an adaptive scheme. Therefore, in this section, we first describe the approach to predict the available bandwidth, and then we propose our segment request strategy which decides the request time and restricts the interval between each request so as to guarantee the synchronization of different live video streams. Based on the results of the two methods discussed above, our adaptive scheduling algorithm is proposed to dynamically adjust the quality levels of multiple video streams and guarantee the quality of multi-view video service under limited and varying bandwidth conditions.

\subsection{Integrated Bandwidth Prediction Approach}

Since there are many existing methods to implement bandwidth prediction, we have improved on a method that utilizes the download throughput samples and adopt it in the approach for its high accuracy [10, 11]. When multiple video streams are requested synchronously in the same transmission cycle, all the requests will get their appropriate segments at once. According to the TCP-Equal share, when both flows compete for the same bottleneck, each uses the same bandwidth[15], so if there are $N$ video streams and the available bandwidth is equal to $W$, then the predicted bandwidth for each request will be $W / N$. In this paper, differ from the researches of TCP in the transport layer[16-19], we only focus on the application layer to design our adaptive scheme. However, we should stay in mind that the concurrent HTTP streaming requests could compete for the bandwidth resource and deteriorate the insufficient bandwidth situation, which is the background of our researches. In this approach, we provide a client-based adaptive frame called shared multi-view adaptive live streaming frame. As shown in Fig.3, all the requests share the same component of bandwidth measurement, segment scheduling and adaptive control. Nevertheless, their buffers are still independent. In this manner, the client can obtain a higher predicted bandwidth, leading to higher request bit rates for the multiple videos.

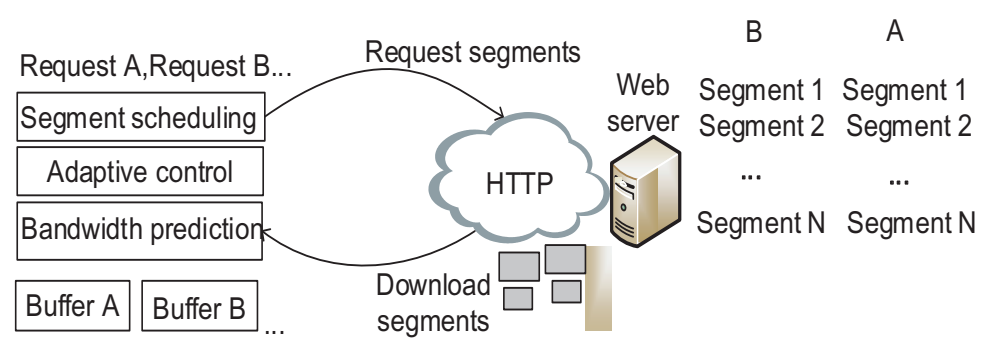

Figure 3: A shared multi-view adaptive live streaming frame

For instance, suppose the available bandwidth is $2 \mathrm{Mbps}$, and the segment length of HAS video streaming is 10 seconds. Video A and video B represent two views of a scenario. Video A has two different bit rates, one is $0.7 \mathrm{Mbps}$ and the other is $1.4 \mathrm{Mbps}$. Video B has only one bit rate, and the bit rate of the stream is $0.5 \mathrm{Mbps}$. When a client treats A and $\mathrm{B}$ as two independent sources and requests them at the same time without the integrated bandwidth prediction, the 
bandwidth predicted for both video $\mathrm{A}$ and video $\mathrm{B}$ is $1 \mathrm{Mbps}$. As a result, the requested bit rates for video $\mathrm{A}$ and video $\mathrm{B}$ will be $0.7 \mathrm{Mbps}$ and $0.5 \mathrm{Mbps}$, respectively. The sizes of the segments for video A and video B will be $7 \mathrm{Mb}$ and $5 \mathrm{Mb}$ accordingly. Sharing the $2 \mathrm{Mbps}$ bandwidth, both video A and video B will have 1Mbps available bandwidth. Therefore, for video $\mathrm{B}$, it takes the client 5 seconds to download the whole $5 \mathrm{Mb}$ segment. In the meantime, video A shares the link with video $\mathrm{B}$, and downloads $5 \mathrm{Mb}$ of the whole segment. After that, video A occupies the $2 \mathrm{Mbps}$ bandwidth exclusively. It takes another 1 seconds to download the remained $2 \mathrm{Mb}$ of the segment. The whole process is shown in Fig.4 (a).

In Fig.4 (b), the integrated bandwidth prediction is adopted. So each request can use the unified measurement of a client to predict the available bandwidth. The unified measurement means that different video requests of a scenario in the same client use the same bandwidth predictor of the client. Therefore, for the same example, the available bandwidth of the client is obtained as $2 \mathrm{Mbps}$. For the client, it is easy to discover that the available bandwidth is sufficient to support video A with 1.4 Mbps and video B with 0.5 Mbps simultaneously. As a result, the requested bit rates for video A and video B will be $1.4 \mathrm{Mbps}$ and $0.5 \mathrm{Mbps}$, respectively. The sizes of the segments for video A and video B will be $14 \mathrm{Mb}$ and $5 \mathrm{Mb}$ accordingly. Sharing the $2 \mathrm{Mbps}$ bandwidth, both video A and video B will also have $1 \mathrm{Mbps}$ available bandwidth. Therefore, for video B, the same as in Fig.4(a), it also takes the client 5 seconds to download the whole segment. In the meantime, video $\mathrm{A}$ also downloads $5 \mathrm{Mb}$ of the whole segment. After that, video A occupies the bandwidth exclusively, and takes another 4.5 seconds to download the remained $9 \mathrm{Mb}$ segment. With the integrated bandwidth prediction, the bit rate of video A can be increased to $1.4 \mathrm{Mbps}$ with higher resource utilization. The whole process is shown in Fig.4(b).

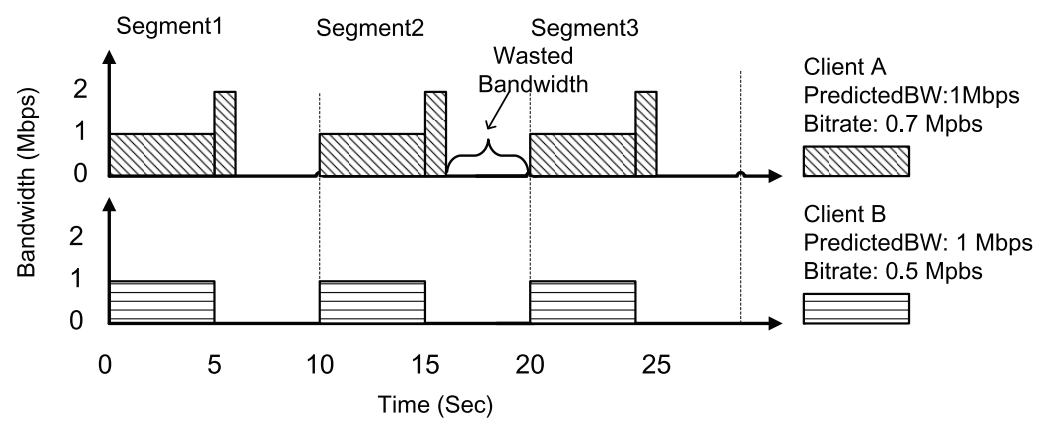

(a) Independent bandwidth prediction.

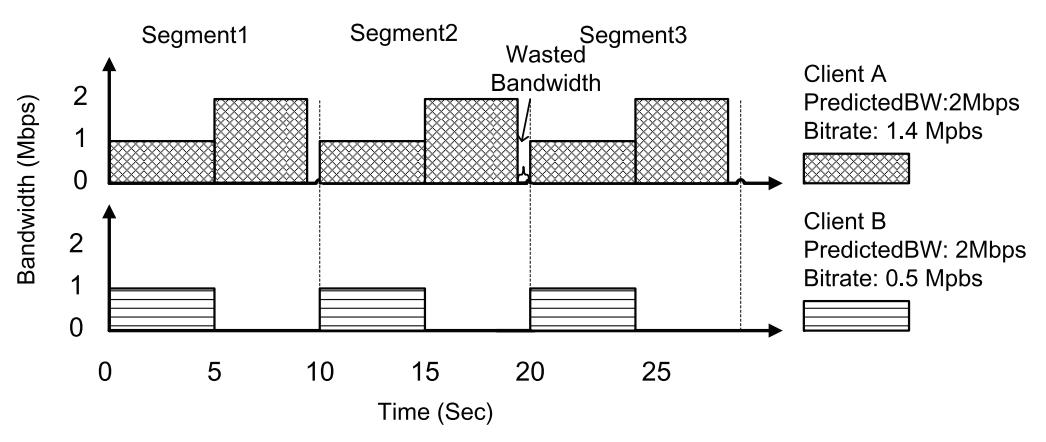

(b) Integrated bandwidth prediction.

Figure 4: An example of Independent and Integrated bandwidth prediction.

\subsection{Unified Segment Request Strategy}

Now our scheme can obtain the available bandwidth with the integrated bandwidth prediction approach discussed above. The strategy in this section is aimed at deciding the time when requests can get their appropriate video segments 
and restrict the interval between each request, so as to play multiple live videos synchronously.

There are mainly two segment scheduling strategies: one is to request the next segment aggressively after the previous segment has been completely downloaded, and the other is to request the segments periodically. The aggressive method fills the buffer of client as quickly as possible, reducing the potential buffer refilling. However, aggressive download may incur redundant data, since the downloaded data will be meaningless when users stop watching the video. Furthermore, the case will be worse when the adaptive scheduling mechanism of HAS is considered. Recent study shows that the redundant data is mainly in conjunction with transient bandwidth upshifts caused by of HAS scheduling rather than the drop out of users[20]. Meanwhile, periodic download scheme tends to maintain a constant playback buffer to avoid unnecessary download. Nowadays, periodic download, combined with some random or aggressive strategy, is broadly adopted. Periodic download works as follows: Let $t_{i+1}^{\text {start }}$ be the time when the client requests segment $i+1, t_{i}^{\text {end }}$ be the time when the transmission of segment $i$ is complete, $\beta(t)$ be the length of the playback buffer (in seconds), $\beta_{t g t}$ be the target buffer which refers to the target length of the playback buffer, $D_{s}$ be the length of each segment, and $T_{d}(i)$ be the length of segment $i$ 's download time. Then, the time to request segment $i+1$ can be written as:

$$
t_{i+1}^{\text {start }}=\left\{\begin{array}{l}
t_{i}^{\text {end }}, \beta(t)<\beta_{t g t} \\
t_{i}^{\text {end }}+D_{s}-T_{d}(i), \beta(t) \geq \beta_{\text {tgt }}
\end{array}\right.
$$

The Equation 1 describes a case that when the predicted bandwidth is less than the sum of all the multiple sources' lowest bit rates, our scheme should suspend the request of the last video stream in the next transmission cycle. We divide the buffer state into the buffer stage and the stable stage according to the size of playback buffer and target buffer: in buffer stage, playback buffer is bigger than target buffer, the client will request the next segment immediately after the previous one has been downloaded completely; in stable stage, the client will request segments periodically, and transmission period is the length of each segment. Our unified segment request strategy for multi-view streaming is based on the periodic download and it can be illustrated as follows.

As we have mentioned before, the video stream which has a higher average bit rate will obtain a higher priority. Let $\mathrm{V}$ denote the set of multiple video streams, which is $\{V(1), V(2) \cdots V(n)\}$ with the average bit rate (i.e., priority) descending order. Let $t_{i+1}^{\text {start }}(n)$ be the time when the client requests segment $i+1$ in $V(n), t_{i}^{\text {end }}(n)$ be the time when the transmission of segment $i$ in $V(n)$ is complete, $\beta^{n}(t)$ be the current data size of $V(n)$ 's buffer, $\beta_{t g t}^{n}$ be the target size of $V(n)$ 's buffer, $D_{s}$ be the length of all the segments, $T_{d}^{n}(i)$ be the length of segment $i$ 's download time in $V(n), t_{i+1}^{g}$ be the time when segment $i+1$ in the server is completely produced, and $\Phi_{i+1}^{n}$ be the quality level of the segment $i+1$ in $V(n)$, then the time to request segment $i+1$ for $V(n)$ can be described as follows.

$$
t_{i+1}^{\text {start }}(n)=\left\{\begin{array}{c}
\max \left(\max \left(t_{i}^{\text {end }}(1), t_{i}^{\text {end }}(2), \cdots, t_{i}^{\text {end }}(n)\right), t_{i+1}^{g}\right), \quad \beta^{n}(t)<\beta_{t g t}^{n} \\
\max \left(\max \left(t_{i}^{\text {end }}(1), t_{i}^{\text {end }}(2), \cdots, t_{i}^{\text {end }}(n)\right)+\max \left(D_{s}-\max \left(T_{d}^{1}(i), T_{d}^{2}(i), \cdots, T_{d}^{n}(i)\right), 0\right), t_{i+1}^{g}\right), \\
\beta^{n}(t) \geq \beta_{t g t}^{n}
\end{array}\right.
$$

Equation 2 describes the strategy about when to request the new segment $i+1$ after previous one $i$. In the stable stage, the client requests segment $i+1$ with the way of periodic request. As shown in Equation 2, the period is equal to the interval of requesting the adjacent precious segment with the highest priority. In this manner, our unified segment request strategy roughly adopts a periodic download strategy to synchronize the multi-view streams by the client. Notice that if the multiple streams are treated independently by the client, it is difficult to exchange the periodic request information among the streams. Meanwhile, in the buffer stage, the aggressive download makes sure that the periodic segment request strategy will not starve the playback buffer. In the buffer stage, playback buffer is smaller than the target buffer. If segment $i+1$ exists, the client requests segment $i+1$ with the way of immediately request, otherwise, the client waits until segment $i+1$ is generated.

Since V is sequenced by the priority descending order, the video stream of the lowest priority is the last one in V. There is a special case at the beginning of this strategy that when the predicted bandwidth is less than the sum of all the multiple sources' lowest bit rates, it must suspend the request of the last video stream in the next transmission cycle; if the bandwidth is still insufficient, the strategy goes on suspending the one before last one. And it maintains this performance until the available bandwidth is able to transmit the rest of the video streams.

When the bandwidth becomes abundant, the suspended requests will be released one after another in the reverse order of suspension, as shown in the following equation. 


$$
t_{i+1}^{\text {start }}(n)=\left\{\begin{array}{l}
\infty, \Phi_{i+1}^{n}=0 \\
t_{i+1}^{\text {start }}(n-1), \text { otherwise }
\end{array}\right.
$$

\subsection{Adaptive Scheduling Algorithm}

With the available bandwidth that is predicted by the approach in Section 4.1, and the time when to request the appropriate segments determined by the strategy in Section 4.2, we can propose an adaptive algorithm for multi-view streaming to further decide the quality level of the required video segments in this part.The proposed multi-view adaptive scheduling algorithm can described as follows:

A. It begins with requesting all the video streams' lowest quality level (i.e., bit rate). When the available bandwidth is less than the sum of all the video streams' lowest bit rate and the data size in the highest priority video's buffer is less than the low threshold (The algorithm similarly adopts two thresholds strategy as many other researches [11, 21]), the algorithm suspends the request with the lowest priority video in the next transmission cycle. If the bandwidth is still insufficient, the algorithm goes on suspending the one before the last one in the sequence until the bandwidth can sustain.

B. On the contrary, if the available bandwidth is more than the sum of all the video streams' lowest bit rates, the algorithm first allows the request of the highest priority video receive a higher bit rate and further increase its quality level if the bandwidth resource is still sufficient. Once the first video stream has already reached its highest bit rate, it increases the second video stream's quality level as well, until the extra bandwidth cannot support the quality improvement any more.

C. During the streaming, if the available bandwidth decreases and becomes less than the sum of all the video streams' current bit rates, the algorithm first allows the lowest priority video decrease its quality level. If the bandwidth is still insufficient, the algorithm further reduces its quality level until it already decreases to its lowest bit rate, then it decreases the previous one as well until the multi-view streaming can meet the network conditions. If all the video streams are decreased to the lowest quality level and the bandwidth is still insufficient, the situation just returns to the initial assumption and deals with it as A.

We set multiple video streams as video $V_{1}$, video $V_{2}$, etc., in the descending order of the priorities. The pseudo-code is shown as Algorithm 1. The time complexity of the algorithm is $O\left(N^{3}\right) . N$ represents the total number of video streams. Since our work focuses on the scenario that the multiple video streams are presented in the same screen, the size of the video number $N$ is usually very small. Therefore, the complexity is acceptable. 


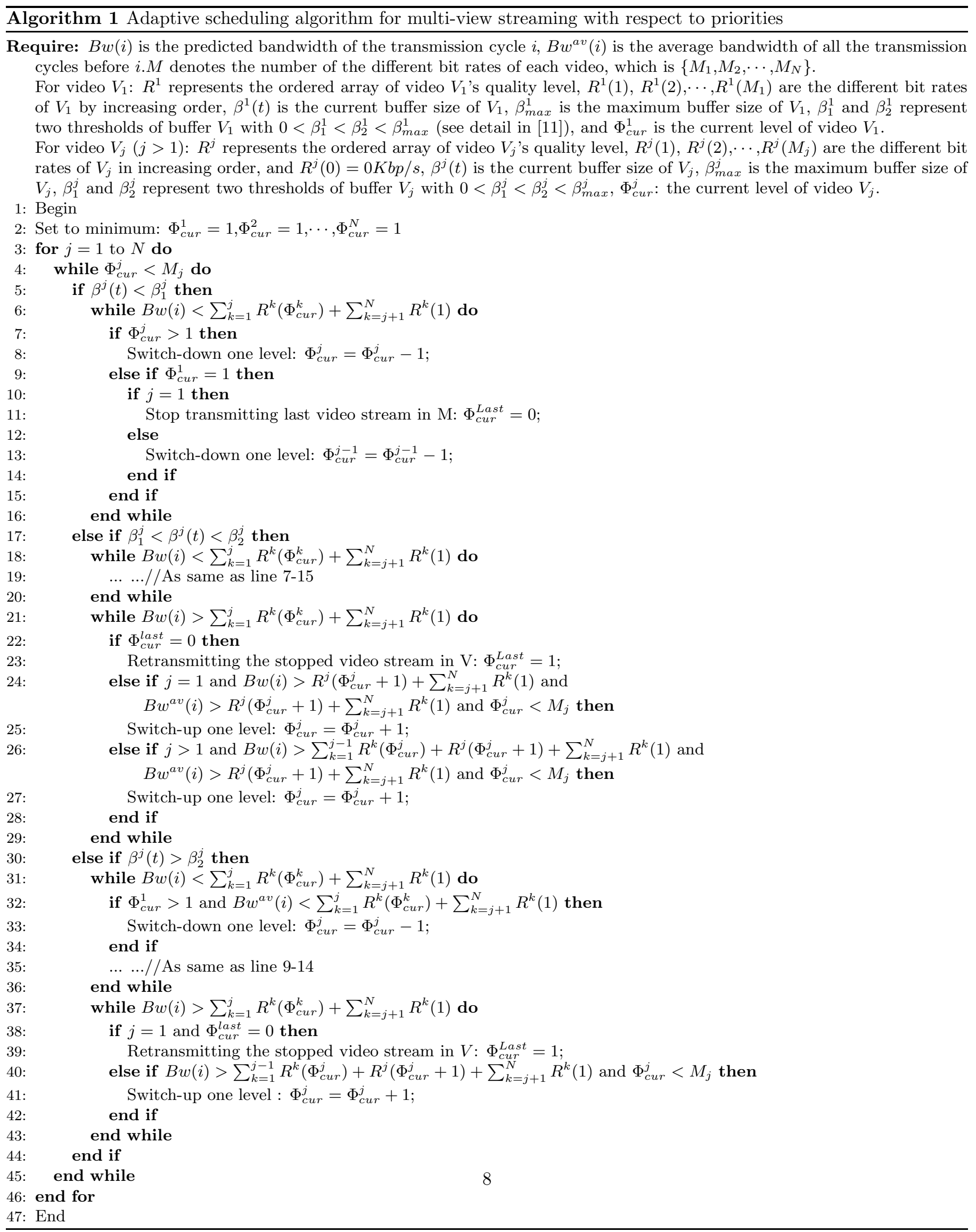




\section{Experiments}

We use the network simulator 2 (NS2) as the experimental simulation environment. Considering the simplicity and rationality, we support two video streams in our experiment. To the best of our knowledge, this is no HAS scheme that is specially designed for multi-view video streams. Therefore, in our simulation, three HAS schemes for single video HTTP adaptive streaming are adopted as the benchmarks. Besides the threshold-based rate adaptation provided by our precious work[11], two highly cited solutions, Festive[12] and Rate adaptation[22], are adopted as the benchmarks to fully demonstrate the efficiency of the proposal. In the simulation, the three benchmarks treat two video streams independently by the client. The simulation results are shown in Fig.5 and Fig.6.

The length of all the video segments is set to 5 seconds. The higher priority video A has four different qualities, and the bit rate is $160 \mathrm{Kbps}, 240 \mathrm{Kbps}, 480 \mathrm{Kbps}$, and $800 \mathrm{Kbps}$, respectively. The lower priority video B has three qualities, and the bit rate is $120 \mathrm{Kbps}, 240 \mathrm{Kbps}$, and $360 \mathrm{Kbps}$, respectively.

\subsection{The Simulation Results of the predicted bandwidth and the Requested Rate}

Firstly, we observe the requested bit rate of these four methods under an unstable network status. The simula-

tion results of the benchmarks are shown in Fig.5(a), Fig.5(b), Fig.5(c), in which the two video streams are treated independently. The result of our multi-view adaptive streaming scheme is shown in Fig.5(d). 


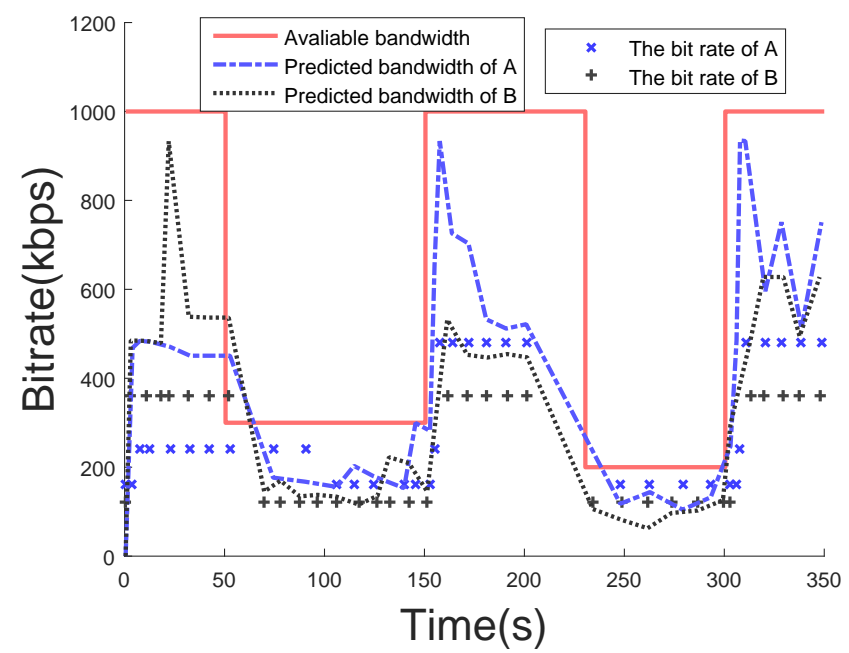

(a) Rate adaptation

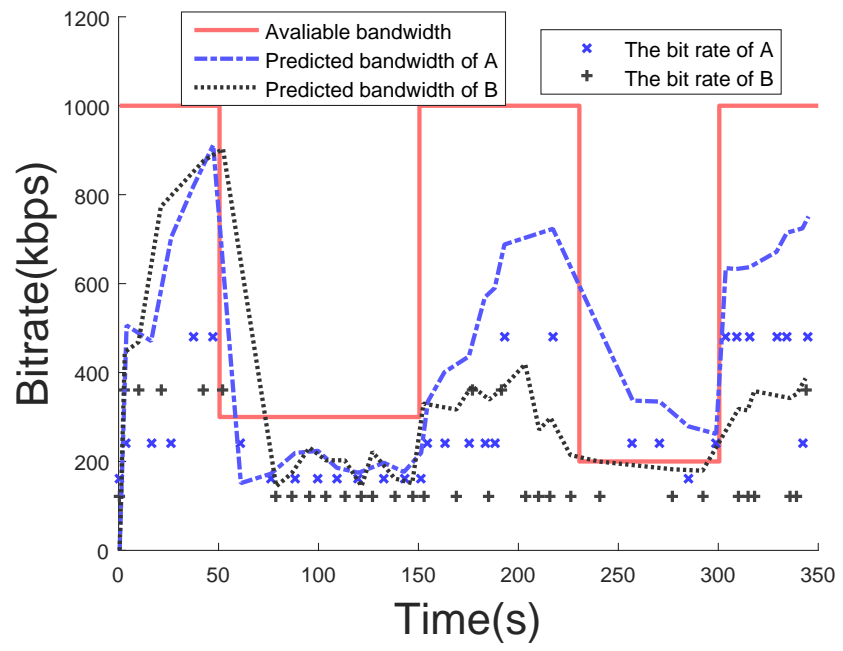

(b) Festive 


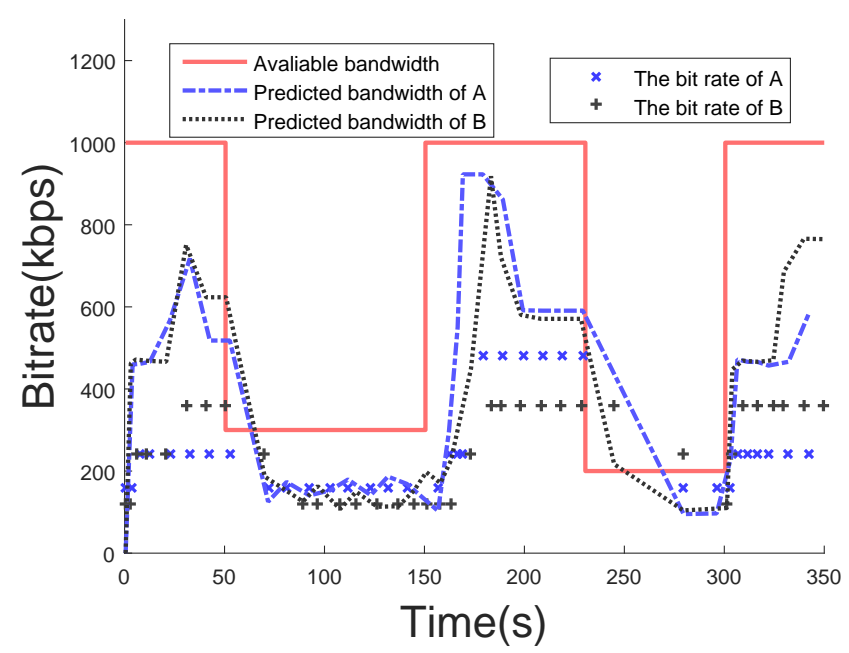

(c) Threshold-based rate adaptation

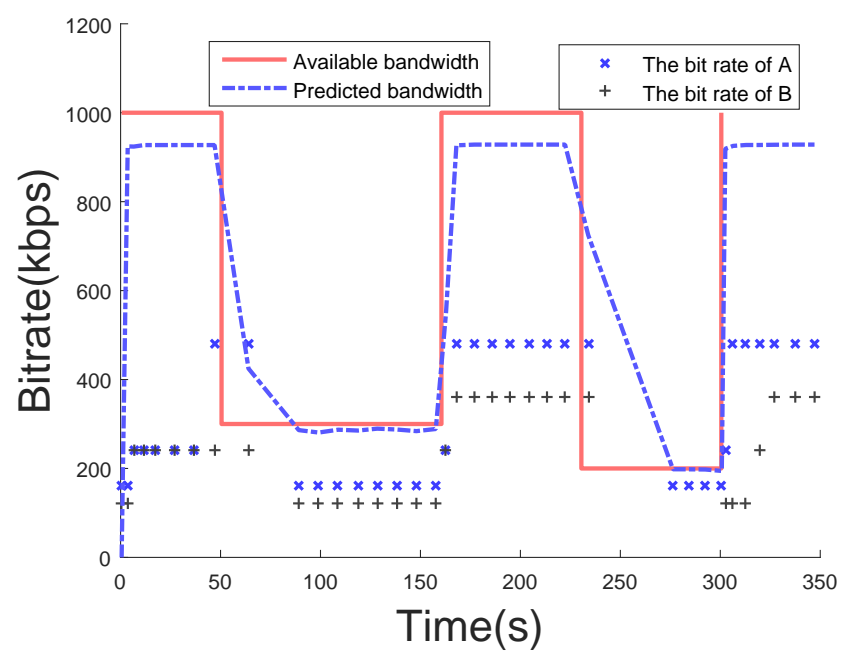

(d) The multi-view adaptive scheme

Figure 5: The simulation results of the request bit rate.

We can see from Fig.5(a), Fig.5(b), Fig.5(c) that if we predict the bandwidth and schedule the multi-view video segments independently, the predicted bandwidth each request can obtain is just about half of the total bandwidth, and the requested video segments are in a lower quality level. From Fig.5(d), we can see that by using our schema, each request obtains the whole bandwidth, so the segments that the receiver requested can be increased to a higher level. Considering the same situation of the available bandwidth, there is no doubt that the utilization rate of the bandwidth is improved. 
Table 1: The results of the average request bit rate and bandwidth utilization.

\begin{tabular}{|c|c|c|c|}
\hline Algorithms & $\begin{array}{c}\text { Average request rate of } \\
\text { video A(Kbps) }\end{array}$ & $\begin{array}{c}\text { Average request rate of } \\
\text { video B(Kbps })\end{array}$ & Bandwidth utilization \\
\hline Rate adaptation & 283.4 & 236.6 & $81.25 \%$ \\
\hline Festive & 288.5 & 178.2 & $72.92 \%$ \\
\hline Threshold-based rate adaptation & 249.1 & 250 & $77.98 \%$ \\
\hline $\begin{array}{c}\text { The multi-view } \\
\text { adaptive scheme }\end{array}$ & 313.5 & 232.7 & $85.34 \%$ \\
\hline
\end{tabular}

Table 1 shows detailed the results of the average request bit rates and the bandwidth utilization results of different methods. From the table, with our method, the requested bit rates of the video with the higher priority are improved significantly with an acceptable decrease in the video with the lower priority. Especially, the multi-view adaptive scheme outperforms the other three benchmarks in terms of the bandwidth utilization. Furthermore, from the figure, segments in the same transmission cycle are requested more simultaneously in our approach compared with the other three methods in Fig.5.

\subsection{The Simulation Results of the receiver's Buffer Size}

The simulation result of the buffer size is shown in Fig.6. The performance of the independent methods are shown in Fig.6(a), Fig.6(b), Fig.6(c), and the performance by the proposed multi-view adaptive scheme is shown in Fig.6(d). 


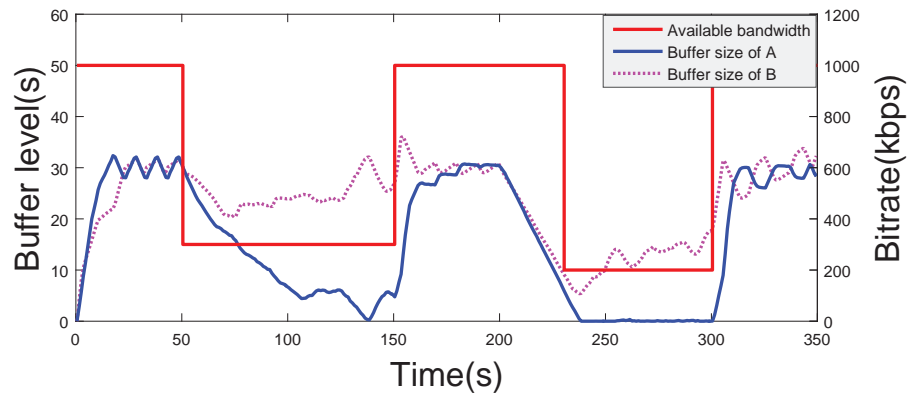

(a) Rate adaptation

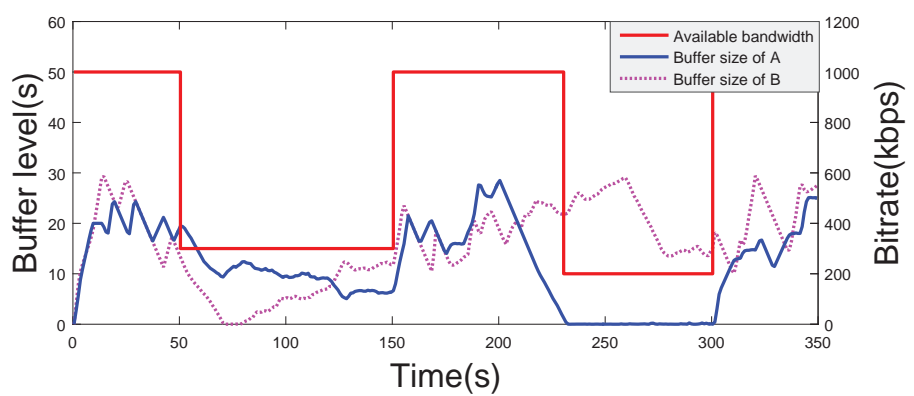

(b) Festive

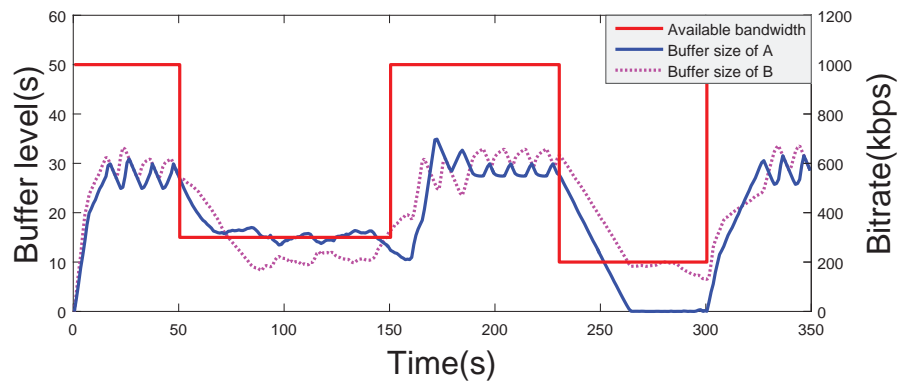

(c) Threshold-based rate adaptation

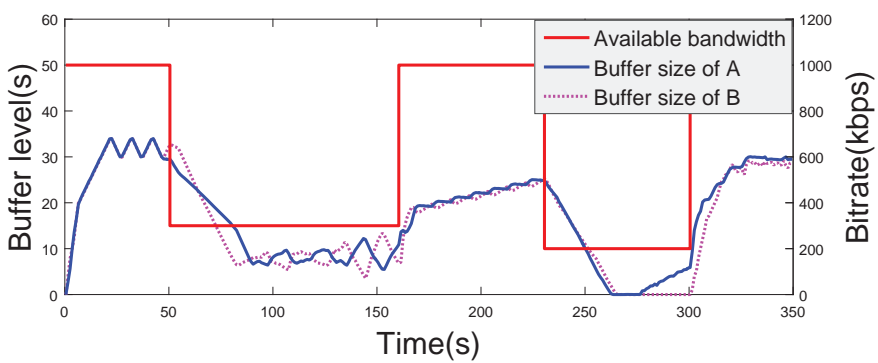

(d) The multi-view adaptive scheme

Figure 6: The simulation results of the buffer size.

Quality of service in video applications is mainly affected by the requested bit rate of video streams. However, many users are willing to tolerate a playback of lower quality video in exchange for the wait or break down during live 
streaming. From Fig.6, we can see that when the available bandwidth descends in $230 \mathrm{~s}-300 \mathrm{~s}$, the buffer size of A by the benchmark suddenly drops to zero and consequently the live streaming is stopped for 37 seconds at least. It harms the user experience severely. But in the proposed multi-view streaming approach, we successfully decrease it to 14 seconds.

\section{Conclusion}

This paper proposes a priority-based adaptive scheme for multi-view live streaming over HTTP, including an integrated bandwidth prediction approach, a unified segment request strategy, and an adaptive scheduling algorithm. The experiment results show that compared with the benchmarks, our scheme can effectively increase the overall bandwidth utilization and provide a synchronous multi-view live streaming. It also can guarantee the quality of high-priority video as well as the overall quality of multi-view video service under limited and varying bandwidth conditions.

\section{Acknowledgements}

This research was partially supported by the National Science Foundation of China under Grant Nos. 61472317, 61428206, 61221063,91118005, 91218301, the MOE Innovation Research Team No. IRT13035, the National Key Technologies R\&D Program of China under Grant No.2013BAK09B01, the Co-ordinator Innovation Project for the Key Lab of Shaanxi Province under Grant No.2013SZS05-Z01, and the China Scholarship Council.

\section{References}

[1] M. Seufert, S. Egger, M. Slanina, T. Zinner, T. Hobfeld, P. Tran-Gia, A survey on quality of experience of http adaptive streaming, IEEE Communication Surveys and Tutorials 17 (2015) $469-492$.

[2] C. Papagianni, A. Leivadeas, S. Papavassiliou, A cloud-oriented content delivery network paradigm: Modeling and assessment, IEEE Transactions on Dependable and Secure Computing 10 (2013) 287-300.

[3] Y. Zhang, N. Ansari, M. Wu, H. Yu, On wide area network optimization, IEEE Communications Surveys and Tutorials 14 (2012) $1090-1113$.

[4] T. Han, N. Ansari, M. Wu, H. Yu, On accelerating content delivery in mobile networks, IEEE Communications Surveys and Tutorials (2013) $1314-1333$.

[5] V. Adzic, H. Kalva, B. Furht, Optimizing video encoding for adaptive streaming over http, IEEE Trans. Consumer Electron. 58 (2012) 397-403.

[6] E. Kurutepe, T. Sikora, Multi-view video streaming over p2p networks with low start-up delay, Proceedings of International Conference on Image Processing (2008) 3088-3091.

[7] W. Zhang, Z. Li, Q. Zheng, Samp: supporting multi-source heterogeneity in mobile p2p iptv system, IEEE Transactions on Consumer Electronics 59 (2013) 772-778.

[8] C. Ozcinar, E. Ekmekcioglu, A. Kondoz, Adaptive 3d multi-view video streaming over p2p networks, in: Proceedings of the IEEE International Conference on Image Processing, IEEE, 2014, pp. 2462-2466.

[9] T. C. Thang, Q. D. Ho, J. W. Kang, A. T. Pnam, Adaptive streaming of audiovisual content using mpeg dash, IEEE Trans. Consumer Electron. 58 (2012) 78-85.

[10] T. C. Thang, A. T. Pham, H. X. Nguyen, P. L. Cuong, J. W. Kang, Video streaming over http with dynamic resource prediction, Proceedings of Communications and Electronics (2012) 130-135.

[11] B. Li, Q. Zheng, W. Zhang, A rate adaptation solution for distance education system over http streaming, Proceeding of 2013 IEEE International Conference on Embedded and Ubiquitous Computing (2013) 2385-2389.

[12] J. Jiang, V. Sekar, H. Zhang, Improving fairness, efficiency, and stability in http-based adaptive video streaming with festive, Proceedings of the 8th International Conference on Emerging Networking Experiments and Technologies (2012) 97-108.

[13] S. C. Son, B. T. Lee, Y. W. Gwak, J. S. Nam, Fast required bandwidth estimation technique for network adaptive streaming, IEEE Trans. Consumer Electron. 56 (2010) 1442-1449.

[14] Y. Liu, S. Yu, J. Zhou, Adaptive segment-based patching scheme for video streaming delivery system, Comput. Commun. 29 (2006) $1889-1895$.

[15] S.-C. Tsao, N. C. T. Univ., Hsinchu, Y.-C. Lai, Y.-D. Lin, Taxonomy and evaluation of tcp-friendly congestion-control schemes on fairness, aggressiveness, and responsiveness, IEEE Communications Society (2007) 6-15.

[16] K. Xu, Y. Tian, N. Ansari, Tcp in wireless environments: Problems and solutions, IEEE Communications Magazine 43 (2005) S27-S32.

[17] Y. Tian, K. Xu, N. Ansari, Tcp-jersey for wireless ip communications, IEEE Journal on Selected Areas in Communications 22 (2004) $747-756$.

[18] H. Nishiyama, N. ansari, N. Kato, Wireless loss-tolerant congestion control protocol designed by dynamic aimd theory, IEEE Wireless Communications 18 (2010) 7-14.

[19] T. Khalifa, A. Abdrabou, K. Naik, M. Alsabaanb, A. Nayak, N. Goel, Split- and aggregated-transmission control protocol (sa-tcp) for smart power grid, IEEE Trans. on Smart Grid 5 (2014) 381-391. 
[20] A. Mansy, M. Ammar, J. Chandrashekar, A. Sheth, Characterizing client behavior of commercial mobile video streaming services, in: Proceedings of Workshop on Mobile Video Delivery, ACM, 2014, p. 8.

[21] S. Akhshabi, S. Narayanaswamy, A. C. Begen, C. Dovrolis, An experimental evaluation of rate-adaptive video players over http, Signal Process.: Image 27 (2012) 271-287.

[22] C. Liu, I. Bouazizi, M. Gabbouj, Rate adaptation for adaptive http streaming, in: Proceedings of the second annual ACM conference on Multimedia systems, ACM, 2011, pp. 169-174. 